\title{
Connective tissue diseases and noninvasive evaluation of atherosclerosis
}

This article was published in the following Dove Press journal: Journal of Vascular Diagnostics

18 June 2014

Number of times this article has been viewed

\author{
Giorgio Ardita \\ Giacomo Failla \\ Paolo Maria Finocchiaro \\ Francesco Mugno \\ Luigi Attanasio \\ Salvatore Timineri \\ Michelangelo Maria Di \\ Salvo
}

Cardiovascular Department, Angiology Unit, Ferrarotto Hospital, Catania, Italy
Correspondence: Giorgio Ardita

Cardiovascular Department, Angiology

Unit, Ferrarotto Hospital, Via Citelli 4,

Catania 95100, Sicily, Italy

Tel +39957436121

Fax +39 957436270

Email giorgio.ardita@tiscali.it
Abstract: Connective tissue diseases (CTDs) are associated with increased risk of cardiovascular disease due to accelerated atherosclerosis. In patients with autoimmune disorders, in addition to traditional risk factors, an immune-mediated inflammatory process of the vasculature seems to contribute to atherogenesis. Several pathogenetic mechanisms have been proposed, including chronic inflammation and immunologic abnormalities, both able to produce vascular damage. Macrovascular atherosclerosis can be noninvasively evaluated by ultrasound measurement of carotid or femoral plaque. Subclinical atherosclerosis can be evaluated by well-established noninvasive techniques which rely on ultrasound detection of carotid intima-media thickness. Flow-mediated vasodilatation and arterial stiffness are considered markers of endothelial dysfunction and subclinical atherosclerosis, respectively, and have been recently found to be impaired early in a wide spectrum of autoimmune diseases. Carotid intima-media thickness turns out to be a leading marker of subclinical atherosclerosis, and many studies recognize its role as a predictor of future vascular events, both in non-CTD individuals and in CTD patients. In rheumatic diseases, flow-mediated dilatation and arterial stiffness prove to be strongly correlated with inflammation, disease damage index, and with subclinical atherosclerosis, although their prognostic role has not yet been conclusively shown. Systemic lupus erythematosus, rheumatoid arthritis, and likely antiphospholipid syndrome are better associated with premature and accelerated atherosclerosis. Inconclusive results were reported in systemic sclerosis.

Keywords: rheumatic disease, subclinical atherosclerosis, arterial stiffness, accelerated atherosclerosis

\section{Introduction}

Cardiovascular (CV) morbidity and mortality in patients with connective tissue diseases (CTDs) are higher than in the general population, primarily due to accelerated atherosclerosis. ${ }^{1}$ However, the mechanisms of enhanced atherogenesis in this population are not yet well defined. Several studies show that traditional CV risk factors such as smoking, hypertension, or diabetes are not sufficient to explain this phenomenon, leading to the assumption of an effect of other nonconventional risk factors. ${ }^{2-4}$ There is growing evidence in favor of persistent inflammation in CTDs, especially in rheumatoid arthritis (RA) and systemic lupus erythematosus (SLE), leading to premature atherosclerosis and its complications. ${ }^{5-7}$ Over time, great emphasis has been placed both on biomarkers of endothelial activation/damage and on noninvasive ultrasound imaging in order to detect early vascular damage and get more accurate risk stratification.

The aim of this review is to summarize aspects of vascular changes found in patients with RA, SLE, antiphospholipid syndrome (APS), and systemic sclerosis (SSc) and, 
when possible, to identify their association with clinically relevant $\mathrm{CV}$ events.

\section{A focus on the problem}

In several studies, SLE and RA seem to be strongly associated with premature and accelerated atherosclerosis and $\mathrm{CV}$ events. Carotid plaques were found with a frequency, ranging between $17 \%$ and $65 \%$ in SLE patients. ${ }^{8,9}$

Data from a multicenter cohort study suggest that in SLE patients, death due to cardiovascular diseases (CVDs) does not appear to be diminished in recent years, in spite of advances in therapies. ${ }^{10}$ SLE patients develop coronary artery disease with a rate from 4 to 8 times higher than normal. ${ }^{11}$ Furthermore, younger SLE patients (age under 45 years) have a 52-fold higher risk of myocardial infarction when compared with matched controls. ${ }^{11}$

Many observational studies, summarized in two reviews including about 100,000 RA patients, showed an increased risk of CVD death (ranging from 50\% to 60\%) when compared with the general population. ${ }^{12,13}$ Notably, subsequent exploratory analyses revealed neither any change in standardized mortality ratios of CV mortality over time nor any relation with disease duration at the time of inclusion, despite advances in treatment in recent decades.

Patients with RA or diabetes have the same increased 3-year rate of fatal and nonfatal CV events when compared with the nondiabetic population, suggesting RA as a risk factor with a CV impact similar to that of diabetes; more precisely, patients with RA and those with type 2 diabetes mellitus had the same hazard ratio when compared with the nondiabetic population. ${ }^{14} \mathrm{~A}$ recent study suggested that $\mathrm{CV}$ risk factors in patients with RA should be targeted as aggressively as in diabetes patients. CV morbidity and mortality in APS and SSc is unclear. There is no conclusive evidence of premature atherosclerosis in APS, ${ }^{15}$ although antiphospholipid antibodies (aPLs) are involved in atherosclerosis pathogenesis. ${ }^{4,16}$

\section{Atherosclerosis and autoimmunity}

Accelerated atherosclerosis in CTDs can be attributed to traditional risk factors for atherosclerosis and use of specific drugs, such as corticosteroids, but also might be the result of other autoimmune and inflammatory mechanisms. ${ }^{15}$

In addition to traditional risk factors, nontraditional risk factors may enhance atherogenesis: systemic inflammation, autoantibodies (aPLs, anti-endothelial cells, anti-double stranded DNA, anti-neutrophil cytoplasmic antibodies), heightened lipid oxidation, heat shock proteins, oxidized low-density lipoprotein/beta2 glycoprotein1 complexes, renal impairment, elevated levels of homocysteine, specific medications (especially steroids), exhaustion of endothelial progenitor cells, and antigenic mimicry mechanisms. ${ }^{2,4,8,15,17,18}$

In SLE and APS, accelerated atherosclerosis may be induced directly by proinflammatory and procoagulant activity of aPLs on endothelial cells or indirectly via the inflammatory/immune mechanisms implicated in autoantibody-mediated thrombosis. ${ }^{16}$ Inconsistently with this notion, Farzaneh-Far et al, ${ }^{19}$ studying 200 SLE patients, found very similar levels of carotid atherosclerosis in aPLs-positive and aPLs-negative patients; moreover, aPLs were not associated with preclinical or clinical atherosclerosis, suggesting that increased cerebrovascular disease in aPLs-positive patients may be better associated with in situ thrombosis or cardiac thromboembolism than with carotid arterial plaque embolization. ${ }^{19}$

Recently, the role of impaired endothelial repair has been highlighted in a study by Rodríguez-Carrio et al, ${ }^{20}$ which showed that angiogenic $\mathrm{T}$ cells and endothelial progenitor cells were strongly decreased in 101 RA patients when compared to matched healthy controls. In a subsequent regression analysis, adjusted for traditional CV risk factors, disease activity, age at diagnosis, antinuclear antibody positivity, and smoking history were shown to be predictive parameters of greater angiogenic $\mathrm{T}$ cell decrease. Finally, a recent review identified type I interferons as predictive biomarkers of endothelial dysfunction in SLE patients. ${ }^{7}$

Therefore, it could be supposed that CTD-related inflammatory and autoimmunity abnormalities, autonomously or synergistically with traditional risk factors, may increase endothelial dysfunction, enhancing atherogenesis.

\section{Methods for noninvasive evaluation of subclinical atherosclerosis}

Macrovascular damage and hemodynamic abnormalities can be noninvasively evaluated by ultrasound measurement of carotid or femoral plaque.

Early subclinical atherosclerosis or impaired arterial stiffness, considered markers of vascular remodeling, can be noninvasively evaluated by ultrasound measurement of carotid intima-media thickness (IMT) and carotid-femoral pulse-wave velocity (PWV), respectively. ${ }^{21-23}$ Indeed, different methods such as augmentation index (Aix) are also known to evaluate carotid-femoral arterial stiffness.

Carotid-femoral PWV is usually obtained at the right common carotid artery and the right femoral artery; it is a direct measurement and corresponds to the widely accepted 
propagative model of the arterial system. Aix explores the retrograde waves proceeding from peripheral arteries, inducing what is called "amplification phenomenon" on aortic walls. ${ }^{22}$ This phenomenon also explains the decreased distensibility and increased pulse pressure usually found in an aging vascular system.

Carotid-femoral PWV and Aix are considered both important markers of systemic arterial stiffness and indicators of the resistance encountered by the left ventricle. ${ }^{24}$

Endothelial dysfunction in CTDs can be noninvasively assessed by the flow-mediated vasodilatation (FMV) method. ${ }^{23}$ It is based on two-dimensional ultrasound detection of the mean diameter and spectral analysis of blood flow in the humeral artery at baseline and after reactive hyperemia is induced by transient occlusive pressure placed around the proximal third of the arm. This method allows for the evaluation of endothelial-dependent vasodilatation. The evaluation is completed by the measurement of endothelium-independent vasodilatation in response to exogenous nitric oxide. ${ }^{25}$

\section{IMT and connective tissue diseases}

Carotid IMT by B-mode ultrasound is the most commonly used method for the detection of subclinical atherosclerosis. A recent systematic review and meta-analysis has shown that carotid IMT can be a useful predictor of future vascular events in otherwise healthy individuals. ${ }^{21}$ In particular, it was shown that for an absolute carotid IMT difference of $0.1 \mathrm{~mm}$, the future risk of myocardial infarction increases by $10 \%$ to $15 \%$, and the stroke risk increases by $13 \%$ to $18 \%$.

In a prospective cohort of patients with SLE, age, cumulative prednisone intake, hypertension, and evidence of antioxidized low-density lipoprotein antibodies were associated with higher IMT. ${ }^{8}$ Some authors report that patients with primary and secondary APS had a higher prevalence of carotid plaque or IMT increase, suggesting a potential independent proatherogenic role for aPLs. ${ }^{16}$

Another systematic review and meta-analysis aimed to assess whether RA, SLE, SSc, and other rheumatic diseases are associated with an increased carotid IMT when compared with healthy control subjects. ${ }^{26}$ In this work, more than 6,800 subjects were selected from about 1,030 studies. A statistically significant greater mean carotid IMT was observed in patients with any rheumatic disease compared with healthy controls, and similarly, there was increased prevalence of plaques in rheumatic diseases. The absolute mean difference in carotid IMT between case and control groups was $0.061 \mathrm{~mm}$ when a random-effects model was applied.
Other investigations provided evidence of increased carotid IMT in RA, and this finding could not be explained by corticosteroid treatment but appeared to be essentially associated with markers of systemic inflammation and disease duration. Of note, higher incidence of vascular events within 5 years is demonstrated in RA patients with increased IMT compared to a similar population without increased IMT. ${ }^{27}$

In RA patients without a history of CV events, carotid ultrasonography, as with IMT and/or carotid plaques, allowed identification of most high/very high CV risk subjects (sensitivity $97.2 \%$ ) much more reliably than the coronary artery calcification score (sensitivity $24 \%$ for a score $>100$ ). ${ }^{28}$

Djokovic et al reported average carotid IMT values higher in SLE patients with aPLs present when compared with SLE patients without aPLs, and in multivariate regression analysis, aPLs were significant predictors of carotid IMT changes in SLE patients. $^{29}$

Inconclusive results were reported in SSc when carotid or femoral IMT were compared with matched controls and healthy controls. With regard to macrovascular involvement, many studies recognized distal peripheral artery disease as the most important vascular involvement in $\mathrm{SSc}^{30}$

\section{Arterial stiffness, FMV, and connective tissue diseases}

Arterial stiffness via PWV adjusted for patients' age and blood pressure was measured in 46 SLE patients. In this study, abnormalities of PWV were associated with traditional and nontraditional CV risk factors. Furthermore, a significant association was found between PWV and the Systemic Lupus International Collaborative Clinics/American College of Rheumatology (SLICC/ACR) damage index score. Multivariate analysis showed that increased PWV was independently associated with metabolic syndrome and SLICC/ ACR damage index score. ${ }^{31} \mathrm{~A}$ recent review ${ }^{32}$ evaluated the relationship in SLE patients between arterial stiffness or endothelial dysfunction and traditional or disease-related risk factors (higher organ damage and activity indexes, longer duration of disease, and raised inflammatory biochemical markers). Higher arterial stiffness was found in patients with traditional CV risk factors and in patients with SLE-related risk factors. It is not clear if impaired FMV is associated with SLE-related risk factors. Nevertheless, the authors highlighted how the prognostic roles of both endothelial dysfunction and arterial stiffness have not yet been well established in appropriate studies. ${ }^{32}$ 
Santos et al used the reactive hyperemia index and Aix (markers of microvascular reactivity and arterial stiffness, respectively) in SLE and RA patients free from CVD and chronic renal dysfunction..$^{33}$ In this study, the authors did not find a significant difference in SLE and RA patients when compared with healthy controls.

In SLE patients with secondary APS, increase in inflammatory markers negatively correlated with endothelialindependent vasodilatation, suggesting how secondary APS affects the vascular smooth muscle and not the endothelium. ${ }^{34}$

In SSc, discrepant results were reported in studies evaluating the relationship among endothelial dysfunction and CV risk factors. ${ }^{30}$

\section{Conclusion}

Several studies have shown that CTDs are associated with higher rates of $\mathrm{CV}$ events; higher rate of $\mathrm{CV}$ events without the presence of conventional risk factors increases suspicion that other mechanisms (likely correlated with the underlying disease) are involved in atherogenesis in autoimmune diseases.

CV morbidity and mortality in patients with CTDs reflect complex and dynamic interaction between traditional risk factors (age, hypertension, metabolic syndrome, diabetes, obesity, tobacco smoking, and cholesterol levels) and CTDrelated risk factors such as organ damage, activity indexes, disease duration, increased inflammatory biochemical markers, adverse or beneficial effects of treatment. Because conventional risk equations are not sufficiently reliable as a result of these complex interactions, it is particularly difficult to adequately assess $\mathrm{CV}$ risk in CTDs patients.

The evaluation of IMT, PWV, Aix, and FMV are proposed as effective methods for noninvasive vascular assessment, especially in early atherogenesis.

Carotid IMT is a leading marker of subclinical atherosclerosis and can be used as a predictor of future vascular events, both in healthy individuals and in CTDs patients. In a number of studies, FMV and arterial stiffness prove to be strongly correlated with inflammation and disease damage index, although their prognostic and predictive value have not yet been conclusively shown. SLE and RA seems to be better associated with premature and accelerated atherosclerosis. Theoretically, primary and secondary APS should be highly related to accelerated atherosclerosis, but it still remains uncertain. Discrepant results were reported when carotid or femoral IMT or endothelial function were measured as surrogate markers of early atherosclerosis in SSc.
New surrogate markers of vascular risk like PWV, Aix, and FMV should be validated for sensitivity, specificity, and predictive value in order to be employed in a more comprehensive screening protocol for $\mathrm{CV}$ risk stratification in rheumatic diseases.

\section{Disclosure}

The authors report no conflicts of interest in this work.

\section{References}

1. Shoenfeld Y, Gerli R, Doria A, et al. Accelerated atherosclerosis in autoimmune rheumatic diseases. Circulation. 2005;112(21):3337-3347.

2. Matsuura E, Kobayashi K, Inoue K, Lopez LR, Shoenfeld Y. Oxidized LDL/beta2-glycoprotein I complexes: new aspects in atherosclerosis. Lupus. 2005;14(9):736-741.

3. Symmons DP, Gabriel SE. Epidemiology of CVD in rheumatic disease, with a focus on RA and SLE. Nat Rev Rheumatol. 2011;7(7):399-408.

4. Lopez LR, Simpson DF, Hurley BL, Matsuura E. OxLDL/beta2GPI complexes and autoantibodies in patients with systemic lupus erythematosus, systemic sclerosis, and antiphospholipid syndrome: pathogenic implications for vascular involvement. Ann N Y Acad Sci. 2005;1051:313-322.

5. Gerli R, Sherer Y, Bocci EB, Vaudo G, Moscatelli S, Shoenfeld Y. Precocious atherosclerosis in rheumatoid arthritis: role of traditional and disease-related cardiovascular risk factors. Ann N Y Acad Sci. 2007;1108:372-381.

6. Bartoloni Bocci E, Luccioli F, Angrisani C, Moscatelli S, Alunno A, Gerli R. Accelerated atherosclerosis in systemic lupus erythematosus and other connective tissue diseases. Expert Rev Clin Immunol. 2007;3(4):531-541.

7. Knight JS, Kaplan MJ. Cardiovascular disease in lupus: insights and updates. Curr Opin Rheumatol. 2013;25(5):597-605.

8. Doria A, Shoenfeld Y, Wu R, et al. Risk factors for subclinical atherosclerosis in a prospective cohort of patients with systemic lupus erythematosus. Ann Rheum Dis. 2003;62(11):1071-1077.

9. Selzer F, Sutton-Tyrrell K, Fitzgerald SG, et al. Comparison of risk factors for vascular disease in the carotid artery and aorta in women with systemic lupus erythematosus. Arthritis Rheum. 2004;50(1):151-159.

10. Bernatsky S, Boivin JF, Joseph L, et al. Mortality in systemic lupus erythematosus. Arthritis Rheum. 2006;54(8):2550-2557.

11. Manzi S, Meilahn EN, Rairie JE, et al. Age-specific incidence rates of myocardial infarction and angina in women with systemic lupus erythematosus: comparison with the Framingham Study. Am J Epidemiol. 1997;145(5):408-415.

12. Aviña-Zubieta JA, Choi HK, Sadatsafavi M, Etminan M, Esdaile JM, Lacaille D. Risk of cardiovascular mortality in patients with rheumatoid arthritis: a meta-analysis of observational studies. Arthritis Rheum. 2008;59(12):1690-1697.

13. Meune C, Touzé E, Trinquart L, Allanore Y. Trends in cardiovascular mortality in patients with rheumatoid arthritis over 50 years: a systematic review and meta-analysis of cohort studies. Rheumatology (Oxford). 2009;48(10):1309-1313.

14. Peters MJ, van Halm VP, Voskuyl AE, et al. Does rheumatoid arthritis equal diabetes mellitus as an independent risk factor for cardiovascular disease? A prospective study. Arthritis Rheum. 2009;61(11):1571-1579.

15. Hollan I, Meroni PL, Ahearn JM, et al. Cardiovascular disease in autoimmune rheumatic diseases. Autoimmun Rev. 2013;12(10):1004-1015.

16. Belizna CC, Richard V, Primard E, et al. Early atheroma in primary and secondary antiphospholipid syndrome: an intrinsic finding. Semin Arthritis Rheum. 2008;37(6):373-380.

17. Shoenfeld Y, Sherer Y, Harats D. Artherosclerosis as an infectious, inflammatory and autoimmune disease. Trends Immunol. 2001;22(6): 293-295. 
18. Blasi C. The autoimmune origin of atherosclerosis. Atherosclerosis 2008;201(1):17-32.

19. Farzaneh-Far A, Roman MJ, Lockshin MD, et al. Relationship of antiphospholipid antibodies to cardiovascular manifestations of systemic lupus erythematosus. Arthritis Rheum. 2006;54(12):3918-3925.

20. Rodríguez-Carrio J, Alperi-López M, López P, Alonso-Castro S, Ballina-García FJ, Suárez A. Angiogenic T cells are decreased in rheumatoid arthritis patients. Ann Rheum Dis. Epub January 7, 2014.

21. Lorenz MW, Markus HS, Bots ML, Rosvall M, Sitzer M. Prediction of clinical cardiovascular events with carotid intima-media thickness: a systematic review and meta-analysis. Circulation. 2007;115(4): 459-467.

22. Laurent S, Cockcroft J, Van Bortel L, et al; European Network for Non-invasive Investigation of Large Arteries. Expert consensus document on arterial stiffness: methodological issues and clinical applications. Eur Heart J. 2006;27(21):2588-2605.

23. Castellon X, Bogdanova V. Screening for subclinical atherosclerosis by noninvasive methods in asymptomatic patients with risk factors. Clin Interv Aging. 2013;8:573-580.

24. Avalos I, Chung CP, Oeser A, et al. Increased augmentation index in rheumatoid arthritis and its relationship to coronary artery atherosclerosis. J Rheumatol. 2007;34(12):2388-2394.

25. Ducharme A, Dupuis J, McNicoll S, Harel F, Tardif JC. Comparison of nitroglycerin lingual spray and sublingual tablet on time of onset and duration of brachial artery vasodilation in normal subjects. Am J Cardiol. 1999;84(8):952-954, A8.

26. Tyrrell PN, Beyene J, Feldman BM, McCrindle BW, Silverman ED, Bradley TJ. Rheumatic disease and carotid intima-media thickness: a systematic review and meta-analysis. Arterioscler Thromb Vasc Biol. 2010;30(5):1014-1026.

27. Gonzalez-Juanatey C, Llorca J, Martin J, Gonzalez-Gay MA. Carotid intima-media thickness predicts the development of cardiovascular events in patients with rheumatoid arthritis. Semin Arthritis Rheum. 2009;38(5):366-371.
28. Corrales A, Parra JA, González-Juanatey C, et al. Cardiovascular risk stratification in rheumatic diseases: carotid ultrasound is more sensitive than Coronary Artery Calcification Score to detect subclinical atherosclerosis in patients with rheumatoid arthritis. Ann Rheum Dis. 2013;72(11):1764-1770.

29. Djokovic A, Stojanovich L, Stanisavljevic N, et al. Does the presence of secondary antiphospholipid syndrome in patients with systemic lupus erythematodes accelerate carotid arteries intima-media thickness changes? Rheumatol Int. Epub November 15, 2013.

30. Hettema ME, Bootsma H, Kallenberg CG. Macrovascular disease and atherosclerosis in SSc. Rheumatology (Oxford). 2008;47(5):578-583.

31. Valero-Gonzalez S, Castejon R, Jimenez-Ortiz C, et al. Increased arterial stiffness is independently associated with metabolic syndrome and damage index in systemic lupus erythematosus patients. Scand $J$ Rheumatol. 2014;43(1):54-58.

32. Chin CW, Tan RS, Thumboo J. Endothelial function and arterial stiffness assessment as early surrogate markers of vascular risk in patients with systemic lupus erythematosus. Clin Exp Rheumatol. 2013;31(2): 295-301.

33. Santos MJ, Carmona-Fernandes D, Canhão H, Canas da Silva J, Fonseca JE, Gil V. Early vascular alterations in SLE and RA patients a step towards understanding the associated cardiovascular risk. PLoS One. 2012;7(9):e44668.

34. Stalc M, Tomsic M, Jezovnik MK, Poredos P. Endothelial-dependent and independent dilation capability of peripheral arteries in patients with systemic lupus erythematosus and antiphospholipid syndrome. Clin Exp Rheumatol. 2011;29(4):616-623.
Journal of Vascular Diagnostics

\section{Publish your work in this journal}

Journal of Vascular Diagnostics is an international, peer-reviewed journal of diagnostics, focusing on non invasive vascular investigation methods involved in the evaluation of vascular diseases. The journal is committed to the rapid publication in the fields of vascular diseases. Original research, review, case reports, expert opinion and commentaries

\section{Dovepress}

are all considered for publication. The manuscript management system is completely online and includes a very quick and fair peer-review system, which is all easy to use. Visit http://www.dovepress.com/testimonials.php to read real quotes from published authors. 